\title{
Escala de estrategias de disposición al estudio en universitarios: propiedades psicométricas ${ }^{1}$
}

\section{Learning Disposition Strategy Scale in University Students: Psychometric Properties}

\author{
Fabiola Sáez-Delgado (1) https://orcid.org/0000-0002-7993-5356 \\ Claudio Bustos Navarrete (2) https://orcid.org/0000-0003-3478-9858 \\ Karla Lobos Peña (2) https://orcid.org/0000-0002-0145-0041 \\ Javier Mella-Norambuena (3) https://orcid.org/0000-0002-4288-142X \\ Alejandro Díaz Mujica (2) http://orcid.org/0000-0002-3090-5463
}

\author{
(1) Universidad Católica de la Santísima Concepción \\ (2) Universidad de Concepción \\ (3) Universidad Técnica Federico Santa María
}

(Recibido: 31 de julio de 2019; Aceptado para su publicación: 17 de enero de 2020)

Cómo citar: Sáez-Delgado, F., Bustos, C., Lobos, K., Mella-Norambuena, J. y Díaz, A. (2021). Escala de estrategias de disposición al estudio en universitarios: propiedades psicométricas. Revista Electrónica de Investigación Educativa, 23, e08, 1-15. https://doi.org/10.24320/redie.2021.23.e08.3253

\section{Resumen}

Las estrategias de disposición al estudio (establecer objetivos, gestionar el tiempo y organizar recursos) son claves para el aprendizaje autorregulado y el desempeño de los estudiantes, por lo que su medición debe ser lo más precisa posible. Sin embargo, los instrumentos para medir estrategias de aprendizaje son muy generales para evaluar estas estrategias disposicionales. Este estudio tuvo como objetivo diseñar y analizar las propiedades psicométricas del instrumento de estrategias de disposición al estudio en estudiantes universitarios, el cual fue aplicado a una muestra por conveniencia de 630 estudiantes universitarios chilenos. Se realizó análisis factorial exploratorio y confirmatorio. La escala total y sus 4 subescalas mostraron consistencia interna adecuada ( $a>0.70$ ), estructura latente ajustada a los datos y correlaciones débiles, aunque estadísticamente significativas, con las calificaciones reportadas por los alumnos. Se concluye que el instrumento es confiable y válido para su aplicación en estudiantes de educación superior.

Palabras clave: planificación de la educación, psicometría, educación superior

\begin{abstract}
Learning disposition strategies (setting goals, managing time, and organizing resources) are key to selfregulated learning and student performance and so they should be measured as accurately as possible. However, instruments to measure learning strategies are too general to evaluate dispositional strategies. The aim of this study was to design and analyze the psychometric properties of a learning disposition strategy instrument for university students, which was administered to a convenience sample of 630 Chilean university students. An explanatory and confirmatory factor analysis was carried out. The full scale

${ }^{1}$ Agradecimiento al Proyecto FONDECYT de Iniciación N¹1201054, financiado por la Agencia Nacional de Investigación y Desarrollo de Chile (ANID).
\end{abstract}


and its four subscales showed adequate internal consistency ( $a>0.70)$, a latent structure that fit the data and weak but statistically significant correlations with student-reported grades. It is concluded that the instrument is reliable and valid for use with higher education students.

Keywords: educational planning, psychometry, higher education

\section{Introducción}

La autorregulación del aprendizaje permite que éste sea autónomo y permanente, asegurando el aumento del rendimiento y éxito académico de los estudiantes especialmente en los primeros años de experiencia universitaria (Broadbent y Poon, 2015; Wolters y Hussain, 2015; Zimmerman y Kitsantas, 2014).

Los modelos de autorregulación del aprendizaje más usados en la investigación teórica y empírica (Boekaerts y Corno, 2005; Hadwin y Oshige, 2011; Pintrich, 2000; Winne y Hadwin, 1998; Zimmerman, 2000) concuerdan en que la autorregulación del aprendizaje es un proceso cíclico que se desarrolla en tres fases: disposición, desempeño y evaluación (Panadero, 2017). De las tres fases la disposición es fundamental, puesto que activa y pone en marcha las siguientes fases del proceso (Durán-Aponte y Pujol, 2013; Mettler et al., 2017).

Las disposiciones al estudio pueden interpretarse como respuestas predeterminadas en presencia de oportunidades y circunstancias de aprendizaje inciertas, donde el estudiante despliega estrategias y creencias para afrontarla (Claxton y Carr, 2004). La disposición de los estudiantes ante la actividad de estudio ayuda en gran medida a explicar los comportamientos de estos sobre su estudio y su proceso de aprender (Tempelaar et al., 2017).

La disposición al estudio se define como las estrategias y creencias que contribuyen a generar actitudes, estados emocionales, motivación y planificación estratégica para llevar a cabo con éxito una tarea académica (Pérez et al., 2011; Sáez et al., 2018; Yan et al., 2014). Este estudio se centra en las estrategias de disposición al estudio correspondiente a: 1) establecer objetivos de estudio, 2) gestionar el tiempo de estudio y 3) organizar tanto los recursos materiales como ambientales (Pérez et al., 2011; Sáez et al., 2018; Zimmerman, 2008).

El establecimiento de objetivos académicos consiste en precisar un propósito de resultados de aprendizaje en un período definido (Dobronyi et al., 2019). La gestión del tiempo académico implica administrar el conocimiento y uso de los espacios temporales necesarios para desarrollar una tarea, y la planificación de ellos para cumplir con los objetivos establecidos (Sayari et al., 2018; Thibodeaux et al., 2017). La organización de recursos es el ordenamiento tanto de los materiales necesarios para atender la tarea de aprendizaje, como el ordenamiento del ambiente para desarrollarla (Puma-at, 2016; Yang et al., 2017).

En educación superior, las estrategias de disposición al estudio son críticas para el éxito académico y constituyen un componente importante del aprendizaje autorregulado, especialmente en estudiantes de primer año (Dresel et al., 2015; Neroni et al., 2019), mostrando múltiples beneficios para los estudiantes: a) determinan la adaptación a la universidad (van Rooij et al., 2017), b) intervienen adecuadamente en la prosecución del estudio y el logro de objetivos académicos (Kolovelonis et al., 2012), c) ayudan a evitar la procrastinación (Iwamoto et al., 2017), d) facilitan la toma de decisiones, consciente e intencional respecto a cómo, cuándo y con quién preparará su estudio (Cetin, 2015), e) permiten control del entorno de estudio, asegurando las mejores condiciones para aprender y alcanzar los mejores resultados (Shernoff et al., 2017), f) mejoran la capacidad para planificar, evaluar, controlar y supervisar su proceso de estudio (Yusuff, 2018), y g) se relacionan positiva y directamente con la implicación en el rendimiento (Neroni et al., 2019; Roick y Ringeisen, 2018).

Es necesario contar con instrumentos válidos y confiables para evaluar el impacto de los programas de promoción de las estrategias de disposición al estudio en universitarios (Chi-Tung y Ruey-Gwo, 2011; Winne, 2010) implementando acciones de intervención educativa basadas en la evidencia disponible (Tempelaar et al., 2017). Una reciente revisión sistemática de la literatura (Roth et al., 2016) mostró que en los 
instrumentos de autoinforme que miden autorregulación del aprendizaje en educación superior faltan escalas para medir disposición al estudio. Si bien existen instrumentos que miden gestión del tiempo, estos no han considerado las otras dos estrategias de disposición que corresponden al establecimiento de objetivos y la organización de recursos, es decir, no se dispone de un instrumento que reúna las tres estrategias de disposición al estudio (Boruchovitch y Dos Santos, 2015; Ramírez-Echeverry et al., 2016).

Considerando la ausencia de instrumentos específicos para evaluar el uso de estrategias de disposición al estudio, el presente trabajo tiene por objetivo analizar el comportamiento psicométrico de un instrumento de medida de estrategias de disposición al estudio en universitarios. Los hallazgos de esta investigación pueden ser relevantes para desarrolladores de programas que buscan ayudar a los estudiantes a mejorar su disposición al estudio para alcanzar las exigencias académicas (Sebesta y Bray, 2017; Triquet et al., 2017), también permitirá contar con una escala para evaluar de forma válida y confiable la efectividad de las intervenciones.

\section{Método}

El estudio consideró un diseño de tipo instrumental, que corresponde a aquellos centrados en la adaptación de pruebas o en la medición de características psicométricas (Ato et al., 2013).

Los participantes de esta investigación fueron 630 estudiantes universitarios y la unidad de muestreo el curso completo. Se realizó un muestreo de tipo no probabilístico por conveniencia, puesto que los participantes correspondían a cursos intactos, dado que por temas administrativos no se podía acceder a los estudiantes de forma individual. Se incluyó a 15 carreras diferentes de 5 universidades del sur de Chile. Del total de la muestra, $48.9 \%$ fueron mujeres $(n=308)$ y el promedio de edad fue de 21.3 años ( $D E=2.69)$. De primer año fueron $23.05 \%$, de segundo año $36.03 \%$, de tercer año $16.14 \%$, de cuarto año $18.60 \%$ y de quinto año $6.18 \%$. Los criterios de inclusión consistieron en que los estudiantes fueran alumnos regulares de una institución de Educación Superior en nivel de pregrado.

Рara medir las tres dimensiones de las estrategias correspondientes a la disposición ante la actividad de estudio (establecimiento de objetivos de estudio, gestión del tiempo de estudio y organización de los recursos tanto materiales como ambientales), se diseñó y validó la Escala de Estrategias de Disposición al Estudio (EEDE) considerando las directrices de la International Test Commission (Muñiz et al., 2013).

La dos primeras subescalas del EEDE son adaptaciones de dos subescalas del Time Management Behavior Scale (TMBQ) (García-Ros y Pérez-González, 2012), específicamente las de establecimiento de objetivo (10 ítems) y gestión del tiempo académico (11 ítems). La tercera escala del EEDE, organización de recursos materiales y ambientales, se construyó seleccionando 10 ítems pertenecientes a 3 instrumentos de estrategias de autorregulación del aprendizaje: 1) Escala de Evaluación de la Autorregulación del Aprendizaje a partir de Textos (ARATEX-R) (Núñez et al., 2015) del cual se utilizaron los ítems 3, 6, 9, 11 y 16; 2) Cuestionario para la Evaluación de las Estrategias de Aprendizaje de los Estudiantes Universitarios (CEVEAPEU) (Gargallo et al., 2009), del cual se utilizaron los ítems 14, 30 y 27; y 3) TMBQ (García-Ros y PérezGonzález, 2012), del cual se utilizaron los ítems 23 y 19. En todos los casos se escribió a los autores de las escalas e ítems originales solicitando su autorización para el desarrollo del instrumento.

La primera versión del EEDE estaba compuesta por 31 ítems (ver Tabla 1). Los ítems 1, 4, 8, 10, 13, 18, 21, 24, 26, 29 correspondían a la subdimensión de establecimiento de objetivos académicos (EOA), los ítems $2,5,7,12,15,17,20,22,25,28$ y 31 a la subdimensión de gestión del tiempo académico (GTA) y los ítems $3,6,9,11,14,16,19,23,27$ y 30 correspondían a la subdimensión de organización de recursos materiales y ambientales (ORMA). Cada pregunta responde a la consigna "con qué frecuencia utilizo la siguiente estrategia" y se contesta en escala de frecuencia ordenada de 5 puntos, donde 1 es "nunca" y 5 es "siempre". A mayor puntuación, mayor disposición al estudio. 
El procedimiento seguido en esta investigación consistió en 3 fases: 1) validación de contenido de la escala por jueces expertos, 2) entrevista cognitiva a estudiantes y 3) aplicación de la escala para el análisis de las propiedades psicométricas.

La primera fase de validación de contenidos por jueces expertos (Urrutia et al., 2014) consistió en contactar a 5 doctores en psicología, 4 chilenos y un español. Cada juez, de forma independiente, respondió una matriz donde se presentó la escala y se les consultó por la suficiencia, claridad y pertinencia de cada ítem (criterio dicotómico adecuado o inadecuado) para medir el constructo estrategias de disposición al estudio y sus 3 dimensiones. Una vez recibidas las matrices de análisis se hizo análisis de Kappa de Cohen, coeficiente que evalúa el nivel de concordancia entre jueces. Los juicios sobre las 3 dimensiones de la escala mostraron un Kappa superior 0.6 en todos los criterios, lo que se considera un nivel alto de consistencia (Cerda y Villarroel, 2008). Se decidió incluir todos los ítems de la escala, ya que hubo acuerdo en que todos eran pertinentes por más de 3 jueces. Se mejoró la redacción en 5 de los ítems que fueron identificados como confusos por más de uno de los jueces.

La segunda fase del estudio fue la entrevista cognitiva a 7 estudiantes universitarios de diferentes carreras y cursos, elegidos en forma intencionada. El objetivo de esta actividad fue identificar problemas de comprensión de instrucciones y redacción de los ítems para obtener evidencias de validez de proceso (Urrutia et al., 2014). Se explicó a los estudiantes el objetivo de la escala y se indicó que podían hacer observaciones sobre la redacción, los contenidos, comprensión de las instrucciones, la forma para responder la escala y la claridad de los ítems. Se solicitó a los estudiantes que con sus propias palabras explicaran a qué se refería cada ítem. Este proceso mostró que la escala es clara y comprensible para los estudiantes.

La última fase correspondió a la aplicación de la escala para analizar las propiedades psicométricas. Primero se invitó a las autoridades de las universidades a participar del estudio, gestionando cartas de autorización para formalizar su compromiso y participación. Los directores de las instituciones que aceptaron participar indicaron en qué carreras se podría realizar el estudio. Se contactó y coordinó la aplicación de la escala con los docentes de las distintas carreras y cursos. De forma preliminar, los docentes informaron a sus estudiantes sobre el objetivo del estudio y la aplicación de las encuestas. El día acordado con el docente se asistió a los cursos y a cada estudiante se le entregó un consentimiento informado, a los que aceptaron participar se les entregó el cuestionario para que lo respondieran. En promedio, el tiempo de respuesta de la escala no fue mayor a 11 minutos.

Antes de comenzar la aplicación del instrumento se solicitó la revisión del protocolo, las cartas de autorización y el consentimiento informado, al Comité de Ética y Bioética de la Universidad de Concepción, Chile. Antes de la aplicación de cada instrumento se aplicó el procedimiento de consentimiento informado, en el cual se comunicaba a los participantes las condiciones del estudio. Cuando estaban de acuerdo con éstas en forma voluntaria se procedió a entregar el cuestionario para su llenado. Se entregó una copia del acta de consentimiento a cada participante.

\subsection{Análisis de los datos}

La muestra $(n=630)$ se dividió en dos porciones iguales, se extrajeron al azar de la población total. En la primera porción ( $n=315)$ se realizó análisis factorial exploratorio (AFE) y en la segunda porción ( $n=315)$, se hizo el análisis factorial confirmatorio (AFC). El análisis de datos consideró primero el análisis descriptivo de la muestra, luego análisis factorial tanto exploratorio como confirmatorio, y por último, se realizó análisis de criterio concurrente con las calificaciones autorreportadas. Todos los análisis se realizaron usando software R versión 3.5 .

Se usó matriz de correlaciones policóricas para realizar AFE debido a que el nivel de medición de los ítems fue de nivel ordinal. Primero, para estudiar la dimensionalidad del constructo se revisaron los índices de KMO y prueba esfericidad de Bartlett explorando la adecuación de la matriz de correlación al análisis factorial (Lloret-Segura et al., 2014). A continuación se realizó análisis paralelo de Horn para definir la cantidad de factores. Iterativamente se fueron eliminando los ítems que presentaban cargas cruzadas o 
bajas comunalidades totales, hasta que finalmente se llegó a una lista de ítems que cargan de forma única en un factor, con cargas superiores a 0.4.

Para confirmar la dimensionalidad de la EEDE se realizó AFC usando estimador WLSMV, adecuado para ítems ordinales. La evaluación del ajuste global del modelo consideró los siguientes estadísticos: Chi cuadrado $\left(x^{2}\right)$, índice de ajuste comparativo (CFI), índice de ajuste no normado (TLI), y el error de aproximación cuadrático medio (RMSEA). Los criterios usados para evaluar adecuadamente el modelo fueron (Hu y Bentler, 1999): a) el x²/gl muestra un buen ajuste con un valor inferior a 3, b) CFI y TLI indica un ajuste aceptable con un valor de 0.90 , y 0.95 , se considera un muy buen ajuste, c) RMSEA con un valor de 0.08 ( $90 \% \mathrm{Cl} 0.10)$ es indicador de un ajuste aceptable, y con 0.05 ( $90 \% \mathrm{Cl} 0.08$ ) es considerado un buen ajuste.

Para el análisis de la confiabilidad de la escala como consistencia interna se utilizó Alfa de Cronbach y Omega. Este último indicador es más adecuado para escalas donde no se cumple el supuesto de tauequivalencia, es decir, aquellos cuyas cargas factoriales son diferentes entre sí (Trizano-Hermosilla y Alvarado, 2016).

Como indicador de validez de criterio concurrente se analizó la correlación entre la escala total y las subescalas con la calificación final del primer semestre y la calificación actual (segundo semestre) autorreportada por los estudiantes.

\section{Resultados}

En la Tabla 1 se muestran los análisis descriptivos de centralidad, dispersión y forma para los ítems de la escala. Se puede observar que los ítems 2, 3, 9, 11, 13 y 19 presentan asimetrías y/o curtosis superiores en valor absoluto a 1. Esto, unido al resultado del test de Mardia que muestran la no normalidad de los datos considerando su asimetría, $p<0.001$, y su curtosis, $p<0.001$, llevan a utilizar la matriz de correlaciones policóricas para la realización del correspondiente análisis factorial. 
Tabla 1. Descriptivos de los ítems de la escala de disposición al estudio

\begin{tabular}{|c|c|c|c|c|c|}
\hline $\mathrm{N}^{\circ}$ & ítems & $M$ & $\mathrm{DE}$ & Asimetría & Curtosis \\
\hline 1 & $\begin{array}{l}\text { Cuando decido lo que intentaré a corto plazo, tengo en cuenta } \\
\text { mis objetivos a largo plazo. }\end{array}$ & 3.68 & 0.89 & -0.27 & -0.33 \\
\hline 2 & $\begin{array}{l}\text { Llevo una agenda conmigo digital o física para organizar mis } \\
\text { actividades. }\end{array}$ & 2.67 & 1.43 & 0.42 & -1.13 \\
\hline 3 & $\begin{array}{l}\text { Me aseguro de tener a mano el material que pueda necesitar } \\
\text { (power point con materia, apuntes, guías de trabajo etc.), antes } \\
\text { de empezar a estudiar. }\end{array}$ & 4.32 & 0.87 & -1.29 & 1.39 \\
\hline 4 & $\begin{array}{l}\text { Repaso mis objetivos académicos para determinar si debo hacer } \\
\text { cambios en mis actividades para lograrlos. }\end{array}$ & 2.84 & 1.05 & 0.16 & -0.29 \\
\hline 5 & $\begin{array}{l}\text { Calculo con precisión el tiempo necesario para cumplir tareas } \\
\text { académicas. }\end{array}$ & 3.13 & 1.10 & -0.03 & -0.66 \\
\hline 6 & $\begin{array}{l}\text { Si hay elementos que me distraen, hago algo para conseguir un } \\
\text { ambiente que permita concentrarme antes de estudiar. }\end{array}$ & 3.70 & 1.05 & -0.49 & -0.49 \\
\hline 7 & $\begin{array}{l}\text { Organizo mis actividades con al menos una semana de } \\
\text { anticipación. }\end{array}$ & 3.03 & 1.07 & -0.01 & -0.60 \\
\hline 8 & Establezco objetivos concretos y evaluables. & 3.35 & 0.94 & -0.18 & -0.28 \\
\hline 9 & $\begin{array}{l}\text { Me aseguro de que el lugar de estudio sea cómodo (luz, } \\
\text { temperatura, ventilación, etc.). }\end{array}$ & 4.22 & 0.89 & -1.07 & 0.77 \\
\hline 10 & Tengo un objetivo claro antes de empezar a estudiar. & 3.96 & 0.92 & -0.73 & 0.33 \\
\hline 11 & $\begin{array}{l}\text { Tengo un cuaderno o block de notas exclusivo para cada } \\
\text { asignatura. }\end{array}$ & 4.12 & 1.28 & -1.28 & 0.29 \\
\hline 12 & Completo las tareas académicas en el horario programado. & 3.57 & 0.95 & -0.34 & -0.33 \\
\hline 13 & Los objetivos que me fijo son realistas y alcanzables. & 4.11 & 0.79 & -0.88 & 1.19 \\
\hline 14 & $\begin{array}{l}\text { Busco maneras de incrementar la eficacia para estudiar } \\
\text { manejando los recursos que dispongo (materiales y ambiente de } \\
\text { estudio). }\end{array}$ & 3.77 & 0.91 & -0.39 & -0.31 \\
\hline 15 & $\begin{array}{l}\text { Diariamente evalúo si estoy cumpliendo con el horario de } \\
\text { estudio preestablecido. }\end{array}$ & 2.56 & 1.12 & 0.3 & -0.63 \\
\hline 16 & $\begin{array}{l}\text { Si tengo algún conflicto familiar o personal busco resolverlo } \\
\text { antes de estudiar para concentrarme. }\end{array}$ & 2.87 & 1.19 & 0.13 & -0.84 \\
\hline 17 & Escribo notas para recordar rápidamente lo que necesito hacer. & 3.29 & 1.26 & -0.24 & -0.93 \\
\hline 18 & $\begin{array}{l}\text { Divido objetivos complejos y difíciles en pequeñas metas más } \\
\text { manejables. }\end{array}$ & 2.91 & 0.99 & -0.09 & -0.42 \\
\hline 19 & Utilizo un sistema de carpetas para organizar la información. & 3.32 & 1.39 & -0.27 & -1.22 \\
\hline 20 & Hago una lista de cosas que hacer cada día. & 2.49 & 1.37 & 0.49 & -0.98 \\
\hline 21 & Diariamente evalúo si estoy cumpliendo con los objetivos fijados. & 2.60 & 1.10 & 0.08 & -0.83 \\
\hline 22 & Establezco un horario de estudio en mi programación diaria. & 2.87 & 1.18 & 0.04 & -0.82 \\
\hline 23 & $\begin{array}{l}\text { Encuentro lugares para estudiar donde puedo evitar } \\
\text { interrupciones y distracciones. }\end{array}$ & 3.89 & 1.03 & -0.81 & 0.05 \\
\hline 24 & $\begin{array}{l}\text { Cumplo mis objetivos académicos antes de realizar actividades } \\
\text { que no son importantes. }\end{array}$ & 3.26 & 0.96 & -0.16 & -0.29 \\
\hline 25 & $\begin{array}{l}\text { Mantengo mi programación, aunque me inviten a otras } \\
\text { actividades. }\end{array}$ & 3.16 & 1.03 & -0.09 & -0.39 \\
\hline 26 & $\begin{array}{l}\text { Establezco objetivos a corto plazo sobre lo que quiero conseguir } \\
\text { en pocos días o semanas. }\end{array}$ & 3.35 & 1.00 & -0.27 & -0.41 \\
\hline 27 & Complemento mis apuntes con los de mis compañeros de clase. & 3.0 & 1.16 & -0.07 & -0.83 \\
\hline 28 & Evito perder tiempo en actividades sin importancia. & 2.94 & 1.11 & 0.20 & -0.71 \\
\hline 29 & Cuando establezco un objetivo considero el plazo para cumplirlo. & 3.47 & 0.99 & -0.22 & -0.53 \\
\hline 30 & $\begin{array}{l}\text { Busco material complementario al entregado en clases para } \\
\text { estudiar (bibliografía del programa de asignatura, libros, } \\
\text { artículos, material audiovisual etc.). }\end{array}$ & 3.42 & 1.13 & -0.27 & -0.83 \\
\hline 31 & $\begin{array}{l}\text { Si sé que voy a tener que esperar un tiempo, preparo alguna } \\
\text { tarea para realizar mientras tanto. }\end{array}$ & 2.93 & 1.09 & 0.09 & -0.54 \\
\hline
\end{tabular}

Nota. Factor Teórico 1. EOA (items: 1, 4, 8, 10, 13, 18, 21, 24, 26, 29); Factor Teórico 2. GTA (items 2, 5, 7, 12, 15, 17, 20, 22, 25, 28, 31); Factor Teórico 3. ORMA (ítems: 3, 6, 9, 11, 14, 16, 19, 23, 27, 30).

\subsection{Análisis Factorial Exploratorio}

Se observó que la matriz de correlaciones policóricas es apta para realizar análisis factorial, ya que obtuvo un KMO de 0.90, y la prueba de esfericidad de Bartlett fue significativa, $x^{2}(465)=4712.8, p<0.001$. Se decidió iniciar el análisis con un modelo de 5 factores, ya que fue el número de componentes a mantener 
indicado por el análisis paralelo de Horn. De forma iterativa, se eliminaron 15 ítems, porque ninguno de ellos lograba tener una carga factorial superior a 0.4 en algún factor. Específicamente, se eliminaron en orden los ítems 10, 22, 12, 21, 4, 18, 15, 3, 31, 5, 16, 11, 30, 27 y 7 . Al eliminar el ítem 18 se elimina el quinto factor, ya que este ítem era el único que cargaba en él, siendo la estructura final de 4 factores.

La solución final de 15 ítems, divididos en 4 factores, explicó un 53\% de la varianza total. En la Tabla 2 se presentan los ítems agrupados a cada factor del instrumento con sus respectivas cargas. El primer factor, que denominaremos Establecimiento de Objetivos Académicos, corresponde a 3 de los 10 ítems del factor del mismo nombre (ej. ítem 20: "Hago una lista de cosas que hacer cada día"). El segundo factor, denominado Gestión del tiempo académico, corresponde a 4 de los 11 ítems del factor original con este mismo nombre (ej. ítem 2: "Llevo una agenda conmigo digital o física para organizar mis actividades"). El tercer factor, llamado Organización de recursos materiales y ambientales, corresponde a 5 de los 10 ítems del factor original (ej: ítem 6: "Si hay elementos que me distraen, hago algo para conseguir un ambiente que permita concentrarme antes de estudiar"). El cuarto factor, orientación a los objetivos, se asocia a estrategias correspondientes al logro de objetivos específicos (ej. ítem 24: "Cumplo mis objetivos académicos antes de realizar actividades que no son importantes").

Tabla 2. Resultado del AFE de la escala de disposición al estudio

\begin{tabular}{|c|c|c|c|c|c|}
\hline Ítem & F1 & F2 & F3 & F4 & $\begin{array}{c}\text { Factor } \\
\text { Original }\end{array}$ \\
\hline \multicolumn{6}{|c|}{ Factor 1. Gestión del Tiempo Académico (GTA) } \\
\hline 20 & 0.80 & -0.01 & 0.07 & 0.00 & GTA \\
\hline 17 & 0.79 & 0.01 & -0.04 & 0.05 & GTA \\
\hline 2 & 0.72 & 0.03 & -0.02 & 0.01 & GTA \\
\hline \multicolumn{6}{|c|}{ Factor 2. Organización de Recursos Materiales y Ambientales (ORMA) } \\
\hline 9 & -0.06 & 0.79 & -0.05 & 0.00 & ORMA \\
\hline 6 & 0.01 & 0.72 & -0.03 & 0.07 & ORMA \\
\hline 23 & 0.08 & 0.67 & 0.12 & -0.04 & ORMA \\
\hline 14 & 0.15 & 0.45 & 0.20 & 0.07 & ORMA \\
\hline \multicolumn{6}{|c|}{ Factor 3. Establecimiento de Objetivos Académicos (EOA) } \\
\hline 29 & 0.09 & -0.05 & 0.62 & 0.11 & EOA \\
\hline 1 & 0.09 & 0.04 & 0.61 & -0.16 & EOA \\
\hline 26 & 0.10 & 0.03 & 0.60 & 0.08 & EOA \\
\hline 8 & -0.05 & 0.15 & 0.59 & 0.08 & EOA \\
\hline 13 & -0.29 & -0.03 & 0.55 & 0.11 & EOA \\
\hline \multicolumn{6}{|c|}{ Factor 4. Orientación a los objetivos (OO) } \\
\hline 24 & -0.01 & 0.04 & -0.02 & 0.86 & EOA \\
\hline 25 & 0.03 & 0.04 & 0.00 & 0.70 & GTA \\
\hline 28 & 0.06 & -0.05 & 0.08 & 0.69 & GTA \\
\hline
\end{tabular}

\subsection{Análisis Factorial Confirmatorio}

Para el AFC se probó con la segunda porción de la muestra el modelo resultante del AFE de 4 factores. El modelo presentó un buen ajuste, ya que si bien el modelo no se ajusta a los datos de forma absoluta, $x^{2}$ $(84)=212.35, p<0.001$, los criterios de ajuste relativo son aceptables: el CFI $=.95$ y TLI $=.93$ presenta un nivel de ajuste superior al valor mínimo recomendado (>0.90) y RMSEA = .067 es menor al nivel máximo recomendado ( $<=0.08$ ). En la Figura 1 se pueden observar las cargas factoriales y correlaciones entre factores del modelo. Todas las cargas factoriales son estadísticamente significativas y se encuentran en el rango .45 a .84. Las correlaciones entre ORMA, EOA y OO son fuertes, en el rango .51 a .57, en tanto que las correlaciones de GTA con los otros 3 factores son moderadas, en el rango . 31 a .32. 
Figura 1. Solución AFC. La numeración de ítems corresponde a la presentada en el Anexo 1

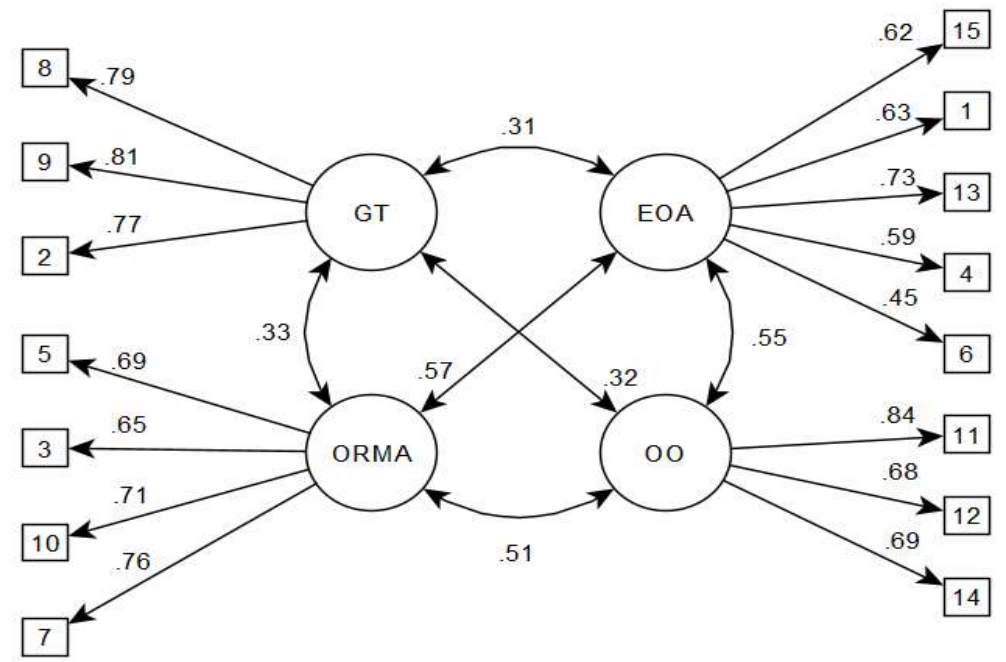

\subsection{Análisis de confiabilidad}

En la Tabla 3 se puede observar que el estadístico Alpha de Cronbach (a) muestra un nivel de confiabilidad aceptable en el AFE y en el AFC para todos los factores (>0.70). Respecto del estadístico Omega $(\omega)$, también muestra un nivel de confiabilidad aceptable (>0.70) en todos los factores del instrumento tanto en el AFE como en el confirmatorio.

Tabla 3. Confiabilidad del Cuestionario de Estrategias de Disposición al Estudio

\begin{tabular}{lllll}
\hline & \multicolumn{2}{c}{ Exploratoria } & \multicolumn{2}{c}{ Confirmatoria } \\
\hline & $\boldsymbol{a}$ & $\boldsymbol{\omega}$ & $\boldsymbol{a}$ & $\boldsymbol{\omega}$ \\
EOA & 0.77 & 0.79 & 0.78 & 0.85 \\
GTA & 0.82 & 0.82 & 0.83 & 0.83 \\
ORMA & 0.80 & 0.81 & 0.79 & 0.81 \\
FE & 0.82 & 0.82 & 0.78 & 0.79 \\
Total & 0.92 & 0.94 & 0.91 & 0.92 \\
\hline
\end{tabular}

Nota. a y w ordinales calculados a partir de la matriz de correlaciones policóricas.

Al correlacionar las subescalas con la calificación obtenida el semestre anterior y la calificación actual reportada por los estudiantes, la cual fue estandarizada tanto por universidad como por carrera, en la muestra total resultó para todos los casos relaciones significativas, aunque la magnitud de las correlaciones fue débil (ver Tabla 4). Específicamente las relaciones entre las diferentes escalas de la EEDE, varían entre $r=0.13$ y $r=0.28$.

Tabla 4. Correlación de Pearson entre estrategias y notas reportadas por estudiantes

\begin{tabular}{lcc}
\hline Estrategia & $\begin{array}{c}\text { Calificación } \\
\text { anterior }\end{array}$ & $\begin{array}{c}\text { Calificación } \\
\text { actual }\end{array}$ \\
\hline Gestión del Tiempo & $0.28^{*}$ & $0.15^{*}$ \\
Organización de Recursos tanto Materiales como Ambientales & $0.15^{* *}$ & $0.13^{*}$ \\
Establecimiento de Objetivos Académicos & $0.18^{* *}$ & $0.20^{* *}$ \\
Orientación a los Objetivos & $0.18^{* *}$ & $0.15^{* *}$ \\
\hline
\end{tabular}

Notas: ${ }^{*} p<0.05 ;{ }^{* *} p<0.01$ 


\section{Discusión y conclusiones}

El propósito de este estudio fue analizar las propiedades psicométricas de la Escala de Estrategias de Disposición al Estudio en estudiantes universitarios chilenos. Se encontró que la prueba cuenta con indicadores de validez de contenido, una estructura ajustada a la teoría, relación significativa con rendimiento académico y niveles buenos de confiabilidad, tanto para la escala general como para cada subescala.

Con respecto a la validez de contenido, ésta fue corroborada en el análisis de jueces expertos y en la aplicación de entrevistas cognitivas a un grupo de estudiantes. Este proceso mostró que los ítems son suficientes, claros y pertinentes para medir disposición al estudio de acuerdo a la evaluación de los jueces expertos y también por la comprensión de los estudiantes respecto de la escala diseñada.

Al estudiar el comportamiento psicométrico del instrumento se encontró que la escala presenta cuatro subdimensiones, de las cuales tres coinciden en las propuestas preliminarmente de forma teórica: establecimiento de objetivos, gestión del tiempo, y organización de recursos tanto materiales como ambientales en el proceso de estudio. La cuarta dimensión que surgió en el proceso de AFE fue orientación a los objetivos. Las cuatro subdimensiones que conforman la escala muestran un Alfa de Cronbach y Omega adecuado, puesto que sus valores son superiores a 0.7 en ambos indicadores. La eliminación de un número importante de ítems en el proceso de AFE se justifica para el logro de una solución simple definida, esto es, con cargas altas en un solo factor (superiores a 0.4) y sin cargas cruzadas, de tal forma que los ítems seleccionados dieran cuenta de forma sólida de cada dimensión. Además, la solución lograda evidencia buenos indicadores de ajuste en el AFC y mostró correlaciones leves, pero significativas estadísticamente, con las calificaciones del semestre anterior y actual que fueron autorreportadas por los estudiantes.

Un resultado interesante es la aparición de una dimensión de orientación a los objetivos, separada del establecimiento de objetivos. El constructo de orientación a los objetivos, si bien no fue considerado de forma preliminar en el diseño del instrumento, es reconocido como relevante en la literatura de la autorregulación del aprendizaje (Kassaw y Astatke, 2017; Pintrich, 2000). La disposición al estudio no sólo depende de la declaración de un objetivo determinado, sino de la focalización para lograrlo, es decir, la orientación hacia el objetivo establecido (Kitsantas et al., 2008).

Este estudio también realizó un análisis de correlación de las cuatro subdimensiones del instrumento con las calificaciones de los estudiantes, en específico se encontraron relaciones significativas, aunque pequeñas en el rango entre $r=0.13$ y $r=0.28$. Este hallazgo es similar a estudios precedentes con universitarios que han mostrado una relación significativa pero pequeña entre el rendimiento académico y la gestión del tiempo (Garzón-Umerenkova y Gil-Flores, 2017; Thibodeaux et al., 2017), establecimiento de objetivos (Dörrenbächer y Perels, 2016; Han y Lu, 2018), organización de recursos materiales y ambientales (Neroni et al., 2019; Schutz et al., 2011) y la orientación a los objetivos académicos (D'Lima et al., 2014; Kassaw y Astatke, 2017).

La EEDE se considera un instrumento con varias fortalezas: a) es una medida fiable y válida; b) demostró en los análisis un adecuado ajuste de sus factores (subdimensiones) y correlaciones apropiadas de los ítems que forman cada una de estas; c) la forma de aplicación es sencilla y rápida; y d) su estructura de cuatro factores brinda una medición específica de las cuatro estrategias de disposición al estudio desplegadas en la primera etapa del proceso de autorregulación del aprendizaje.

Las estrategias de disposición desplegadas en la fase inicial del proceso de autorregulación del aprendizaje han mostrado en estudios previos su relación directa con el desempeño académico de los estudiantes, especialmente en primer año de universidad. Por lo tanto, contar con una escala de medición de estas estrategias claves para el éxito de las exigencias de la educación superior de estudiantes novatos permitiría identificar de forma temprana niveles inadecuados de desarrollo de éstas para iniciar acciones que permitan fomentar las estrategias de disposición en los estudiantes. 
Además, el diseño y validación de este tipo de escalas podría ser muy útil para investigadores que buscan evaluar tanto el impacto de intervenciones para la promoción y mejora de los procesos de autorregulación del aprendizaje, además de analizar la relación de estas estrategias con otras variables académicas importantes en el éxito de los estudios.

Entre las limitaciones de esta investigación, se incluyen las calificaciones consideradas para los análisis, por una parte fueron autorreportadas por los estudiantes, por lo que podría existir un sesgo respecto de las calificaciones formales que son presentadas en los registros académicos. Por otro lado, en el caso de las calificaciones correspondientes al semestre actual, fueron parciales y no finales, desconociéndose la calificación lograda al término del semestre académico.

En futuros estudios se sugiere realizar un análisis de la relación de las diferentes dimensiones de la escala con calificaciones formales recuperadas del registro académico formal de los estudiantes, y no con calificaciones autorreportadas. Se sugiere también analizar empíricamente la pertinencia del uso de la escala en diferentes grupos de estudiantes universitarios, de varias carreras de distintas disciplinas, universidades y años de estudio en Educación Superior.

\section{Referencias}

Ato, M., López, J. y Benavente, A. (2013). Un sistema de clasificación de los diseños de investigación en psicología. Anales de Psicología, 29(3), 1038-1059. https://doi.org/10.6018/analesps.29.3.178511

Boekaerts, M. y Corno, L. (2005). Self-regulation in the classroom: a perspective on assessment and intervention. Applied Psychology, 54(2), 199-231. https://doi.org/10.1111//.1464-0597.2005.00205.x

Boruchovitch, E. y Dos Santos, A. (2015). Psychometric studies of the learning strategies scale for university students. Paidéia, 25(60), 19-27. https://doi.org/10.1590/1982-43272560201504

Broadbent, J. y Poon, W. (2015). Self-regulated learning strategies y academic achievement in online higher education learning environments: A systematic review. The Internet and Higher Education, 27, 1-13. https://doi.org/10.1016/j.iheduc.2015.04.007

Cerda, J. y Villarroel, L. (2008). Evaluación de la concordancia inter-observador en investigación pediátrica: Coeficiente de Kappa. Revista Chilena de Pediatría, 79(1), 54-58. https://doi.org/10.4067/S0370$\underline{41062008000100008}$

Cetin, B. (2015). Academic motivation and self-regulated learning in predicting academic achievement in college. Journal of International Education Research, 11(2), 95-106. https://doi.org/10.19030/jier.v11i2.9190

Chi-Tung, C. y Ruey-Gwo, C. (2011). The construction and verification of a self-regulated learning process model of the electrical technology basic competency. Learning, 5(19), 862-870.

Claxton, G. y Carr, M. (2004). A framework for teaching learning: the dynamics of disposition. Early Years, 24(1), 87-97. https://doi.org/10.1080/09575140320001790898

D'Lima, G., Winsler, A. y Kitsantas, A. (2014). Ethnic and gender differences in first-year college students' goal orientation, self-efficacy, and extrinsic and intrinsic motivation. The Journal of Educational Research, 107(5), 341-356. https://doi.org/10.1080/00220671.2013.823366

Dobronyi, C., Oreopoulos, P. y Petronijevic, U. (2019). Goal setting, academic reminders, and college success: A large-scale field experiment. Journal of Research on Educational Effectiveness, 12(1), 38-66. https://doi.org/10.1080/19345747.2018.1517849 
Dörrenbächer, L. y Perels, F. (2016). Self-regulated learning profiles in college students: their relationship to achievement, personality, and the effectiveness of an intervention to foster self-regulated learning. Learning and Individual Differences, 51(1), 229-241. https://doi.org/10.1016/i.lindif.2016.09.015

Dresel, M., Schmitz, B., Schober, B., Spiel, C., Ziegler, A., Engelschalk, T., Jöstl, G., Klung, J., Roth, A., Wimmer, B. y Steuer, G. (2015). Competencies for successful self-regulated learning in higher education: structural model and indications drawn from expert interviews. Studies in Higher Education, 40(3), 454-470. https://doi.org/10.1080/03075079.2015.1004236

Durán-Aponte, E. y Pujol, L. (2013). Manejo del tiempo académico en jóvenes que inician estudios en la Universidad Simón Bolívar. Revista Latinoamericana de Ciencias Sociales, 11(1), 93-108.

http://revistaumanizales.cinde.org.co/rlcsnj/index.php/Revista-Latinoamericana/article/view/835

García-Ros, R. y Pérez-González, F. (2012). Spanish version of the time management behavior questionnaire for university students. The Spanish Journal of Psychology, 15(3), 1485-1494.

https://doi.org/10.5209/rev SJOP.2012.v15.n3.39432

Gargallo, B., Suarez-Rodríguez, J. y Pérez-Pérez, C. (2009). El Cuestionario CEVEAPEU. Un instrumento para la evaluación de las estrategias de aprendizaje de los estudiantes universitarios. Relieve, 15(2), 1-31.

https://doi.org/10.7203/relieve.15.2.4156

Garzón-Umerenkova, A. y Gil-Flores, J. (2017). Gestión del tiempo en alumnado universitario con diferentes niveles de rendimiento académico. Educação e Pesquisa, 44(1), 1-16. https://doi.org/10.1590/s1678$\underline{4634201708157900}$

Hadwin, A. y Oshige, M. (2011). Self-regulation, coregulation, and socially shared regulation: exploring perspectives of social in self-regulated learning theory. Teachers College Record, 113(2), 240-264.

Han, J. y Lu, Q. (2018). A correlation study among achievement motivation, goal-setting and L2 learning strategy in EFL context. English Language Teaching, 11(2), 5-14. https://doi.org/10.5539/elt.v11n2p5

Hu, L. y Bentler, P. (1999). Cutoff criteria for fit indexes in covariance structure analysis: Conventional criteria versus new alternatives. Structural Equation Modeling: A Multidisciplinary Journal, 6(1), 1-55.

https://doi.org/10.1080/10705519909540118

Iwamoto, D., Hargis, J., Bordner, R. y Chandler, I. (2017). Self-regulated learning as a critical attribute for successful teaching and learning. International Journal for the Scholarship of Teaching and Learning, 11(2), 110. https://doi.org/10.20429/ijsotl.2017.110207

Kassaw, K. y Astatke, M. (2017). Gender, academic self-efficacy, and goal orientation as predictors of academic achievement. Global Journal of Human-Social Science Research, 17(6), 55-65.

Kitsantas, A., Winsler, A. y Huie, F. (2008). Self-Regulation and Ability Predictors of Academic Success During College: A Predictive Validity Study. Journal of Advanced Academics, 20(1), 42-68.

Kolovelonis, A., Goudas, M. y Dermitzaki, I. (2012). The effects of self-talk and goal setting on self-regulation of learning a new motor skill in physical education. International Journal of Sport and Exercise Psychology, 10(3), 221-235. https://doi.org/10.1080/1612197X.2012.671592

Lloret-Segura, S., Ferreres-Traver, A., Hernández-Baeza, A. y Tomás-Marco, I. (2014). El análisis factorial exploratorio de los ítems: una guía práctica, revisada y actualizada. Anales de Psicología, 30(3), 1151-1169. https://doi.org/10.6018/analesps.30.3.199361 
Mettler, J., Carsley, D., Joly, M. y Heath, N. (2017). Dispositional mindfulness and adjustment to university. Journal of College Student Retention: Research, Theory \& Practice, 21(1), 38-52.

https://doi.org/10.1177/1521025116688905

Muñiz, J., Elosua, P. y Hambleton, K. (2013). Directrices para la traducción y adaptación de los tests: segunda edición. Psicothema, 25(2), 151-157. https://doi.org/10.7334/psicothema2013.24

Neroni, J., Meijs, C., Gijselaers, H., Kirschner, P. y de Groot, R. (2019). Learning strategies and academic performance in distance education. Learning and Individual Differences, 73(4), 1-7.

https://doi.org/10.1016/j.lindif.2019.04.007

Núñez, J., Amieiro, N., Álvarez, D., García, T. y Dobarro, A. (2015). Escala de evaluación de la autorregulación del aprendizaje a partir de textos (ARATEX-R). European Journal of Education and Psychology, 8(1), 9-22. https://doi.org/10.1016/j.ejeps.2015.10.002

Panadero, E. (2017). A review of self-regulated learning: six models and four directions for research. Frontiers in Psychology, 8, 1-28. https://doi.org/10.3389/fpsyg.2017.00422

Pérez, M., Valenzuela, M., Díaz, A., González-Pienda, J. y Núñez, J. (2011). Disposición y enfoques de aprendizaje en estudiantes universitarios de primer año. Universitas Psychologica, 10(2), 441-449.

file:///C:/Users/sopor/Downloads/691-Texto\%20del\%20art\%C3\%ADculo-5616-1-10-20111012.pdF

Pintrich, P. (2000). The role of goal orientation in self-regulated learning. En M. Boekaerts, P. Pintrich, y M. Zeidner (Eds.), Handbook of Self-Regulation (pp. 451-502). Academic Press. https://doi.org/10.1016/B978$\underline{012109890-2 / 50043-3}$

Puma-at, R. (2016). Study habits of business students towards achieving academic performance. International Journal of Liberal Arts and Social Science, 4(5), 57-70.

Ramírez-Echeverry, J., García-Carrillo, A. y Olarted, F. (2016). Adaptation and validation of the motivated strategies for learning questionnaire -MSLQ- in engineering students in Colombia. International Journal of Engineering Education, 32(4), 1774-1787.

Roick, J. y Ringeisen, T. (2018). Students' math performance in higher education: Examining the role of selfregulated learning and self-efficacy. Learning and Individual Differences, 65, 148-158.

https://doi.org/10.1016/i.lindif.2018.05.018

Roth, A., Ogrin, S. y Schmitz, B. (2016). Assessing self-regulated learning in higher education: A systematic literature review of self-report instruments. Educational Assessment, Evaluation and Accountability, 28(3), 225-250. https://doi.org/10.1007/s11092-015-9229-2

Sáez, F., Bustos, C., Pérez, M., Mella, J., Lobos, K. y Díaz, A. (2018). Disposición al estudio, autoeficacia y atribuciones causales en estudiantes universitarios chilenos. Propósitos y Representaciones, 6(1), 1-18.

http://dx.doi.org/10.20511/pyr2018.v6n1.179

Sayari, K., Jalagat, R. y van Dalluay, M. (2018). Assessing the relationship of time management and academic performance of the business students in Al-Zahra college for women. European Business y Management, 3(1), 1-8. https://doi.org/10.11648/j.ebm.20170301.11

Schutz, C. M., Gallagher, M. L. y Tepe, R. E. (2011). Differences in learning and study strategies inventory scores between chiropractic students with lower and higher grade point averages. The Journal of Chiropractic Education, 25(1), 5-10. 
Sebesta, A. y Bray, E. (2017). How should I study for the exam? Self-regulated learning strategies and achievement in introductory biology. CBE Life Sciences Education, 16(2), 1-12.

https://doi.org/10.1187/cbe.16-09-0269

Shernoff, D. J., Ruzek, E. y Sinha, S. (2017). The influence of the high school classroom environment on learning as mediated by student engagement. School Psychology International, 38(2), 201-218.

https://doi.org/10.1177/0143034316666413

Tempelaar, D., Rienties, B. y Nguyen, Q. (2017). Towards actionable learning analytics using dispositions. IEEE Transactions on Learning Technologies, 10(1), 6-16.

Thibodeaux, J., Deutsch, A., Kitsantas, A. y Winsler, A. (2017). Firsty-year college students' time use: Relations with self- regulation and GPA. Journal of Advanced Academics, 28(1), 5-27.

https://doi.org/10.1177/1932202X16676860

Triquet, K., Peeters, J y Lombaerts, K. (September 2017). Self-regulated learning online: Empirical foundations, promotion and evaluation for teacher professional Development. Contributing SRL Part to TeachUp. A policy experimentation co-funded by Erasmus+. Deliverable D1:1: Gaps in ITE and CPD provision report. Department of Educational Sciences, Vrije Universiteit Brussel.

Trizano-Hermosilla, I. y Alvarado, J. (2016). Best alternatives to Cronbach's alpha reliability in realistic conditions: Congeneric and asymmetrical measurements. Frontiers in Psychology, 7(1), 1-8.

https://doi.org/10.3389/Fpsyg.2016.00769

Urrutia, M., Barrios, S., Gutiérrez, M. y Mayorga, M. (2014). Métodos óptimos para determinar validez de contenido. Revista Cubana de Educacion Médica Superior, 28(3), 547-558.

http://www.ems.sld.cu/index.php/ems/article/view/301

van Rooij, E., Jansen, E. y van de Grift, W. (2017). First-year university students' academic success: the importance of academic adjustment. European Journal of Psychology of Education, 33, 749-767.

https://doi.org/10.1007/s10212-017-0347-8

Winne, P. (2010). Improving measurements of self-regulated learning. Educational Psychologist, 45(4), 267276. https://doi.org/10.1080/00461520.2010.517150

Winne, P. y Hadwin, A. (1998). Studying as self-regulated learning. En D. J. Hacker, J. Dunlosky, y A. C. Graesser (Eds.). Metacognition in educational theory and practice (pp. 277-304). Routledge.

https://doi.org/10.4324/9781410602350

Wolters, C. y Hussain, M. (2015). Investigating grit and its relations with college students' self-regulated learning and academic achievement. Metacognition and Learning, 10(3), 293-311.

https://doi.org/10.1007/s11409-014-9128-9

Yan, V., Thai, K.-P. y Bjork, R. (2014). Habits and beliefs that guide self-regulated learning: do they vary with mindset? Journal of Applied Research in Memory and Cognition, 3(3), 140-152.

https://doi.org/10.1016/j.jarmac.2014.04.003

Yang, G., Badri, M., Al Rashedi, A., Almazroui, K., Qalyoubi, R. y Nai, P. (2017). The effects of classroom and school environments on student engagement: the case of high school students in Abu Dhabi public schools. Compare: A Journal of Comparative and International Education, 47(2), 223-239.

https://doi.org/10.1080/03057925.2016.1230833

Yusuff, K. (2018). Does personalized goal setting and study planning improve academic performance and perception of learning experience in a developing setting? Journal of Taibah University Medical Sciences, 13(3), 232-237. https://doi.org/10.1016/j.jtumed.2018.02.001 
Zimmerman, B. (2000). Attaining self-regulation a social cognitive perspective. En M. Boekaerts, P. Pintrich, y M. Zeidner (Eds.), Handbook of Self-Regulation (pp. 13-40). Academic Press. https://doi.org/10.1016/B978$\underline{012109890-2 / 50031-7}$

Zimmerman, B. (2008). Investigating self-regulation and motivation: Historical background, methodological developments, and future prospects. American Educational Research Journal, 45(1), 166183. https://doi.org/10.3102/0002831207312909

Zimmerman, B. y Kitsantas, A. (2014). Comparing students' self-discipline and self-regulation measures and their prediction of academic achievement. Contemporary Educational Psychology, 39(2), 145-155.

https://doi.org/10.1016/i.cedpsych.2014.03.004 
Anexo 1.

\section{Escala de Estrategias de Disposición al Estudio (EEDE)}

El propósito de este instrumento es conocer el uso de estrategias de disposición al estudio en estudiantes universitarios.

En esta escala no existen respuestas correctas ni incorrectas. Responda de la manera más honesta posible, por favor. Encierra en un círculo tus respuestas.

Responda a la siguiente consigna: ¿Con qué frecuencia utilizo las siguientes estrategias?

Tabla A. Formato de respuesta de la Escala de Estrategias de Disposición al Estudio

\begin{tabular}{|c|c|c|c|c|c|c|}
\hline & $\begin{array}{l}\text { Responde teniendo en cuenta esta } \\
\text { asignatura }\end{array}$ & Nunca & $\begin{array}{l}\text { Pocas } \\
\text { veces }\end{array}$ & $\begin{array}{l}\text { Algunas } \\
\text { veces }\end{array}$ & $\begin{array}{l}\text { Muchas } \\
\text { veces }\end{array}$ & Siempre \\
\hline $\begin{array}{c}1 \\
(1) \\
2\end{array}$ & $\begin{array}{l}\text { Cuando decido lo que intentaré a corto plazo } \\
\text { tengo en cuenta mis objetivos a largo plazo. }\end{array}$ & 1 & 2 & 3 & 4 & 5 \\
\hline $\begin{array}{l}(2) \\
3\end{array}$ & $\begin{array}{l}\text { para organizar mis actividades. } \\
\text { Si hay elementos que me distraen, hago algo }\end{array}$ & 1 & 2 & 3 & 4 & 5 \\
\hline (6) & $\begin{array}{l}\text { para conseguir un ambiente que permita } \\
\text { concentrarme antes de estudiar. }\end{array}$ & 1 & 2 & 3 & 4 & 5 \\
\hline $\begin{array}{c}4 \\
(8)\end{array}$ & Establezco objetivos concretos y evaluables. & 1 & 2 & 3 & 4 & 5 \\
\hline $\begin{array}{l}5 \\
(9) \\
6\end{array}$ & $\begin{array}{l}\text { Me aseguro de que el lugar de estudio sea } \\
\text { cómodo (luz, temperatura, ventilación, etc.). } \\
\text { Los objetivos que me fijo son realistas y }\end{array}$ & 1 & 2 & 3 & 4 & 5 \\
\hline $\begin{array}{l}(13) \\
7\end{array}$ & $\begin{array}{l}\text { alcanzables. } \\
\text { Busco maneras de incrementar la eficacia }\end{array}$ & 1 & 2 & 3 & 4 & 5 \\
\hline (14) & $\begin{array}{l}\text { para estudiar manejando los recursos que } \\
\text { dispongo (materiales y ambiente de estudio). }\end{array}$ & 1 & 2 & 3 & 4 & 5 \\
\hline $\begin{array}{c}8 \\
(17) \\
9\end{array}$ & $\begin{array}{l}\text { Escribo notas para recordar rápidamente lo } \\
\text { que necesito hacer. } \\
\text { Hago una lista de cosas que hacer cada día. }\end{array}$ & 1 & 2 & 3 & 4 & 5 \\
\hline (20) & & 1 & 2 & 3 & 4 & 5 \\
\hline $\begin{array}{l}10 \\
(23) \\
11\end{array}$ & $\begin{array}{l}\text { Encuentro lugares para estudiar donde } \\
\text { puedo evitar interrupciones y distracciones. } \\
\text { Cumplo mis objetivos académicos antes de }\end{array}$ & 1 & 2 & 3 & 4 & 5 \\
\hline $\begin{array}{l}(24) \\
12\end{array}$ & $\begin{array}{l}\text { realizar actividades que no son importantes. } \\
\text { Mantengo mi programación, aunque me }\end{array}$ & 1 & 2 & 3 & 4 & 5 \\
\hline $\begin{array}{l}(25) \\
13\end{array}$ & $\begin{array}{l}\text { inviten a otras actividades. } \\
\text { Establezco objetivos a corto plazo sobre lo }\end{array}$ & 1 & 2 & 3 & 4 & 5 \\
\hline (26) & $\begin{array}{l}\text { que quiero conseguir en pocos días o } \\
\text { semanas. }\end{array}$ & 1 & 2 & 3 & 4 & 5 \\
\hline $\begin{array}{l}14 \\
(28) \\
15\end{array}$ & $\begin{array}{l}\text { Evito perder tiempo en actividades sin } \\
\text { importancia. } \\
\text { Cuando establezco un objetivo considero el }\end{array}$ & 1 & 2 & 3 & 4 & 5 \\
\hline (29) & plazo para cumplirlo. & 1 & 2 & 3 & 4 & 5 \\
\hline
\end{tabular}

Nota: La primera columna muestra la nueva numeración correlativa de los ítems desde el 1 al 15, mientras que los números entre paréntesis en la misma columna corresponden al número que tenía el ítem en el AFE. 